\title{
Glass-Fiber Woven Catalysts as Alternative Catalytic Materials for Various Industries. A Review
}

\author{
V. V. Barelko ${ }^{a, b}$, M. V. Kuznetsov ${ }^{c, *}$, V. G. Dorokhov ${ }^{a}$, and I. Parkin ${ }^{d}$ \\ ${ }^{a}$ Institute of Problems of Chemical Physics, Russian Academy of Sciences, Chernogolovka, Moscow oblast, 142432 Russia \\ ${ }^{b}$ OOO "Khimfist", Chernogolovka, Moscow oblast, 142432 Russia \\ ${ }^{c}$ All-Russia Scientific Research Institute on Problems of Civil Defense and Emergency Situations Federal \\ (Federal Science and High Technology Center), Ministry of the Russian Federation on Civil Defense, \\ Emergency Situations, and Response to Accidents' Implications, Moscow, 121352 Russia \\ ${ }^{d}$ Materials Chemistry Research Center, Department of Chemistry, University College London, \\ London, WC1H OAJ United Kingdom \\ *e-mail:maxim1968@mail.ru
}

\begin{abstract}
The chemistry and technology of new versatile multipurpose catalytic systems developed and studied by the authors for the purposes of heterogeneous catalysis are reviewed. A theoretical background for a successful search for these new catalytic systems is based on an unconventional approach with emphasis on an essential role of branched-chain reaction mechanisms of heterogeneous catalysis previously developed by the authoring team. The catalytic systems under study are based on silica (aluminoborosilicate) glass-fiber amorphous matrices doped with various metals and manufactured as articles with various types of woven structure. The specific features of these glass-fiber woven catalytic systems, such as their structure, phase state of the matrix, manufacture and activation methods, design of catalytic reactors in which they operate, as well as production technologies and operation methods, make a compelling case to regard them as a new separate class of catalysts. As compared to conventional catalytic materials, these new catalysts are highly efficient in neutralizing industrial gas emissions, in contact stages of the production of nitric acid and sulfuric acid, in various reactions of catalytic hydrocarbon processing, in water purification from nitrate and nitrite contaminants, in catalytic heat generation, etc.
\end{abstract}

Keywords: heterogeneous catalysis, glass-fiber woven catalysts, silica-based catalysts, aluminoborosilicate catalysts, cassette-type design, catalytic reactor, practical applications

\section{INTRODUCTION}

The technological developments considered in this review were initiated and further developed based on a concept unconventional for heterogeneous catalysis, according to which branched-chain, energetically nonequilibrium stages of self-activation of the catalyst surface play an important role in the catalytic mechanisms. The basic assumptions of the concept were formulated in the early 1970 s and then extended to introduce into the theory of heterogeneous catalytic reactions new ideas on the stability of catalytic processes to spatially localized perturbations, autowave rearrangements of the operation modes of catalytic reactors, and phenomena of domain instability arising in such systems [1]. Previously, the authoring team under the guidance of Professor V.V. Barelko carried out a series of studies that revealed the nature of the anomalously high migration mobility of active sites on the catalyst surface and the mechanisms of loss of stability in catalytic systems. The results of these investigations have provided the basis for searching for approaches to the synthesis and design of new types of materials for catalysis and adsorption, and for the creation of new principles of catalytic reactor engineering.

\section{SILICA GLASS-FIBER WOVEN CATALYSTS (GENERAL INFORMATION)}

This type of wide-use catalytic systems was created while solving a specific technological problem, namely in searching for means to suppress the surface migration mobility and sublimation loss of platinum from the catalyst in catalytic combustion reactions, particularly in the ammonia conversion stage in the production of nitric acid. Catalytic systems with the parameters required for these processes have been created based on silica glass-fiber woven materials, activated by implanting the ions of a number of metals into the glass-fiber amorphous matrix of the support. Subsequent laboratory studies and industrial tests have 


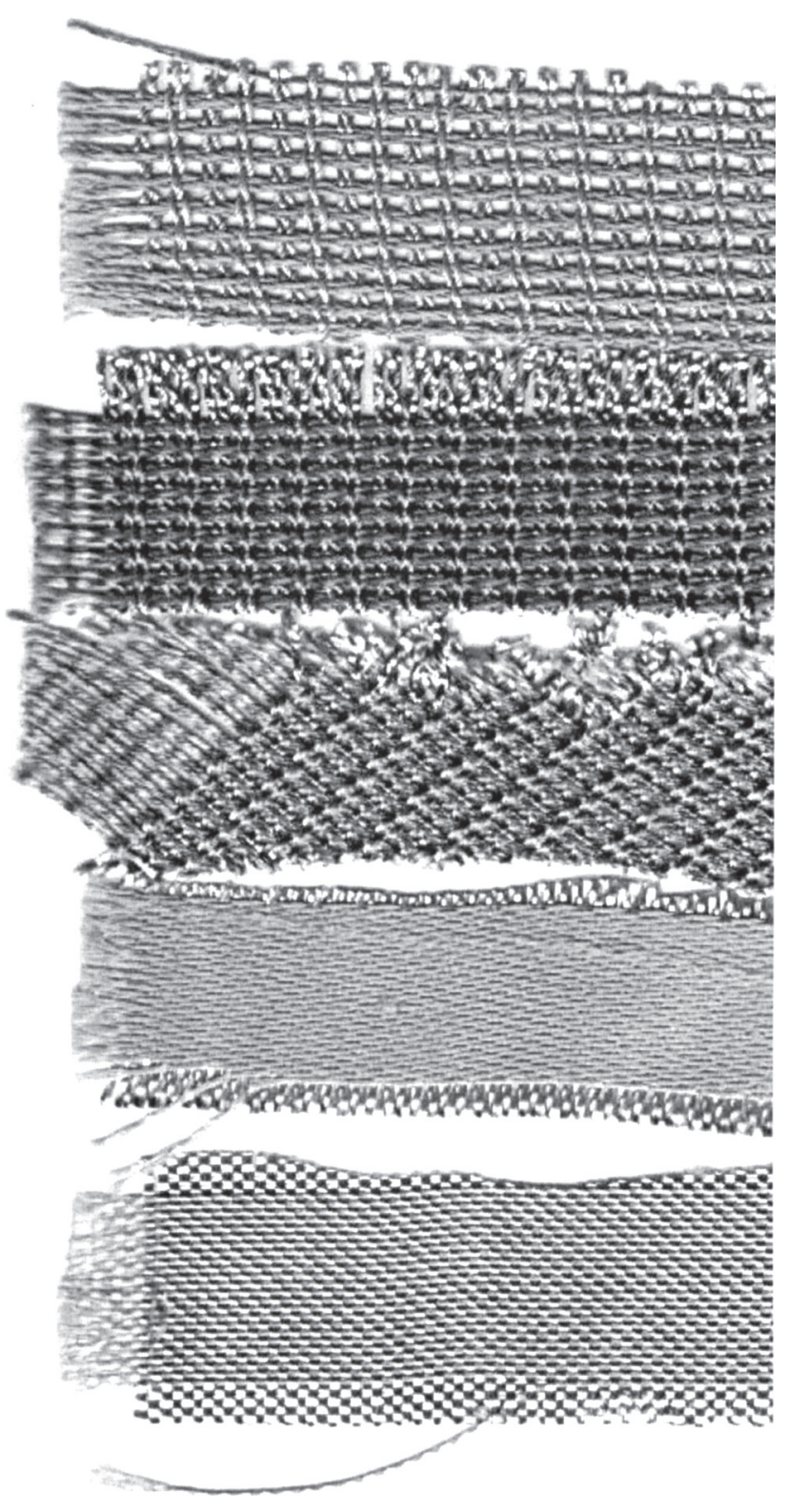

Fig. 1. Woven glass-fiber catalysts of various structures activated by metals; from top to bottom: $\mathrm{Cr}, \mathrm{Pd}, \mathrm{Pt}, \mathrm{Cr}$, and Pt. The sizes of the samples: $\sim 150 \mathrm{~mm}$ in length and $\sim 60 \mathrm{~mm}$ in width.

shown that such catalytic systems can be very effective in many existent and newly developed technological processes. It can be expected that silica glass-fiber woven catalysts (SGFWCs) are able to replace traditional powdered and granular bulk catalytic materials in technological practice.

The SGFWC element is an article woven of silicate, amorphous-phase glass fibers $\left(\mathrm{SiO}_{2}\right.$ content, 55-98 wt \%) in the form of fabrics or networks activated by catalytic components from a wide variety of metals (Pt, Pd, Ag, Cr, Ni, Mn, Co, etc.). Their chemical composition and percentage are determined by the requirements of each individual catalytic process. Figure 1 shows images of these materials, illustrating the unusual appearance and design of SGFWCs (various woven-pattern samples doped with platinum, palladium, and chromium) [2-4].

The modern technology of production of glassfiber materials also makes it possible to manufacture SGFWC elements in the form of three-dimensional blocks with a very high degree of regularity of their geometric structure. This property of the catalytic glass-fiber woven block, which is an alternative to expensive and brittle honeycomb ceramic blocks, seems very important from the point of view of the enhancement of the thermal stability of exothermic catalytic processes, achievable by suppressing technologically dangerous "domain instability" and local overheatings in catalytic reactors, so-called "hotspots". In search for means of the catalytic activation of the silica (aluminoborsilicate) base of SGFWCs, a number of methods for implanting metal ions into the amorphous glass-fiber matrix of SGFWCs during their manufacture have been developed. The most promising is the method based on ion-exchange reactions that occur directly in the microporous solid amorphous matrix of the SGFWC placed in a special liquid medium containing metal ions.

Historically, our research on such catalysts (SGFWCs) is pioneering. Before our publications, there was no information on any systematic studies of catalysis on amorphous silica glass-fiber (or aluminoborsilicate) matrices. Some attempts to use silica woven materials in manufacturing catalysts for neutralizing automobile exhaust gases were undertaken in the 1970 s and 1980s. In this respect, it is worthwhile to mention the patents published in these years, which described engineering solutions relating to catalysts based on silica woven materials [5-7]. However, in these proposals, a glass-fiber woven material was exclusively used as a support for a metallic active phase, which was applied onto its surface by conventional methods. Issues relating to the intrinsic catalytic properties of amorphous glass-fiber silica matrices, as well as the possibility of their catalytic activation by targeted doping with metals, have not even been addressed in these patents.

Let us single out the main factors that determine the principle scientific and technological novelty of SGFWC systems and impart to these systems significant advantages in terms of catalytic and operational characteristics as compared to traditional bulk powder and granular catalysts:

- SGFWCs are formed on glass-fiber amorphous silica (aluminoborosilicate) matrices. Such a base, characterized by phase metastability, is unconventional for classical catalysis on silica supports (silica gels), which relies on crystalline (polycrystalline) materials or materials the phase state of which is not certified. The phase disequilibrium of the base of SGFWC systems is their principal feature, since it is this 
factor that largely determines the possibility of manifestation of unusual catalytic properties of SGFWCs.

- The catalytic activation of SGFWCs is carried out by the implantation of metal ions into an amorphous silica matrix. As a result of this operation, the glassy matrix acquires unusual catalytic properties, with the element introduced into its structure (metal in a reduced or oxidized state) being firmly retained there, remaining unaffected by migration, agglomeration, and separation from the carrier. This method of activation of the silica matrix, based on its doping with metals, is unconventional for classical catalysis. In classical catalytic reactions, catalytically active metalcontaining phases are deposited on the support, a procedure that cannot fully ensure the chemical, phase, and mechanical unity of the support-catalytically active phase system.

- SGFWC elements are characterized by a high catalytic activity at a very small content of the metal component (hundredth-tenths of percent by weight) in the matrix. This feature of SGFWC systems, emerging due to the catalytic activation of the entire inert silica matrix by doping with metal ions, seems especially important for developing a wide variety of catalysts with an active phase prepared from various noble metals.

- SGFWC elements are characterized by a very developed catalytic surface. Macroporosity is determined and regulated by selecting a specific multi-fiber structure of the matrix, which is formed by twisting individual fibers with a diameter of several microns into a working thread and by weaving threads into a working fabric (simple fabric, twill, satin, mesh, jacquard weaving, etc.). The microporosity of SGFWCs is easily regulated by changing the chemical composition of the glass and by performing special pretreatment of the glass-fiber support matrix. The internal specific surface area of SGFWCs can vary, according to the requirements of the specific catalytic process, from units (for alkali glass) to hundreds (for aluminoborosilicate glass) of square meters per gram of catalyst, with a wide pore size distribution $(10-1000 \AA)$.

-SGFWCs are characterized by high chemical and thermal stability, mechanical strength, resistance to abrasion and dusting. These properties of SGFWCs, in conjunction with the stability of the metal component introduced into their matrix, provide a long service life of these systems.

- the use of SGFWCs in technological processes makes it possible to realize an effective cassette-type design in the reactor due to a layer-by-layer packing of the catalyst bed, which leads to a significant reduction in the total mass of the charge compared to conventional bulk powder or granular catalysts. Such a cassette-type design of the catalyst cartridge makes its installation and extraction (when spent) easy.

- the process of production of SGFWC materials is characterized by the continuity of the technological scheme, its easy retuning to a new product, and economy. The creation of catalyst factories for the produc- tion of SGFWC materials does not require significant capital investments, since they can be launched by introducing some additional stages into already existing production of glass-fiber materials for heat-shielding, electrical insulating, and construction purposes.

Thus, SGFWCs can be classified as new, almost unexplored objects of catalytic chemistry. Catalytic systems of this type are characterized by fundamental scientific novelty and the novelty of their technological applications. The main distinguishing features of SGFWC systems as a new technical solution are protected by the RF patent [8]. Unclassified information on these catalysts and their practical applications was first given in $[9,10]$. At present, the results of the research on SGFWCs and their applications in various technological processes are presented in a rather large number of publications, with the technical solutions found being protected by domestic and foreign patents.

At present, the production of industry batches of glass-fiber catalysts has already been mastered. The potential annual production is estimated as $1000000 \mathrm{~m}^{2}$ (500-700 tons) of catalyst fabric. The optimization of the regimes of the industrial process, formulation of technological regulations for the production of catalysts, and carrying out of pilot and industrial-scale tests are conducted by a small innovative enterprise OOO "Khimfist" in cooperation of a number of research organizations and industrial enterprises. The currently identified industry branches of use of this class of SGFWCs are as follows:

(1) Catalytic purification of industrial gas emissions from organic impurities, $\mathrm{CO}$, and nitrogen oxides.

(2) Production of nitric acid and fertilizers.

(3) Production of sulfuric acid.

(4) Various processes of hydrogenation of hydrocarbons:

(4.1) hydrogenation of nitroaromatic hydrocarbons, in particular for the purpose of utilizing explosives;

(4.2) selective hydrogenation of acetylenic impurities in synthesis gases in the production of olefins and synthetic rubber monomers;

(4.3) hydrogenation of natural oils and fats in the production of hydrogenated fats (margarine industry and production of technical solid fats).

(5) Purification of water from nitrate-nitrite contaminations by hydrogenation.

(6) Catalytic surface nitriding of items made of steels and alloys in mechanical engineering.

In the following sections, brief information is given on the status and prospects of the development of SGFWCs in some of these industry branches. 
Table 1. Comparative characteristics of SGFWC systems and traditional granular $\mathrm{Al}_{2} \mathrm{O}_{3}$-based catalysts in the process of purification of industrial gas emissions

\begin{tabular}{l|l|l|c|c|c|c|c}
\hline \multicolumn{1}{c|}{ System } & $\begin{array}{c}\text { Weight } \\
\text { of loading }\end{array}$ & $\begin{array}{c}\text { Form } \\
\text { of loading }\end{array}$ & $\begin{array}{c}\text { Duration } \\
\text { of installation } \\
\text { and } \\
\text { service life } \\
\text { replacement } \\
\text { of catalytic } \\
\text { material }\end{array}$ & $\begin{array}{c}\text { Temperature } \\
\text { at catalysis } \\
\text { zone inlet }\end{array}$ & $\begin{array}{c}\text { Cost pegree } \\
\text { of purification } \\
\text { of purification } \\
\text { system } \\
\text { (relative units) }\end{array}$ \\
\hline $\begin{array}{l}\mathrm{Al}_{2} \mathrm{O}_{3} \text {-based tra- } \\
\text { ditional granular } \\
\text { catalys }\end{array}$ & $>1 \mathrm{t}$ & $\begin{array}{l}\text { Bulk-density } \\
\text { layer }\end{array}$ & $10000 \mathrm{~h}$ & $\begin{array}{l}2-3 \text { working } \\
\text { shift }\end{array}$ & $350-400^{\circ} \mathrm{C}$ & $90 \%$ & 1 \\
\hline \begin{tabular}{l} 
SGFWC system \\
\hline
\end{tabular} & $60-80 \mathrm{~kg}$ & $\begin{array}{l}\text { Catalytic } \\
\text { cassette }\end{array}$ & $(12-15) \times 10^{3} \mathrm{~h}$ & $1-2 \mathrm{~h}$ & $*$ & $>90 \%$ & 0.3 \\
\hline
\end{tabular}

\section{SGFWC SYSTEMS FOR THE CATALYTIC PURIFICATION OF INDUSTRIAL GAS EMISSIONS CONTAINING ORGANIC POLLUTION}

The authors propose new systems for the catalytic purification of gas emissions from organic pollutants. These systems can be used in the machine-building, automotive, and cable industry, furniture manufacturing, and any other industry to neutralize gas emissions in the air outflowing from paint and varnish lines and installations. The proposed process is realized using SGFWC materials activated by noble metals, with the content of the latter in the SGFWC matrix of $0.05-0.2 \mathrm{wt} \%$. As already pointed out earlier, the structure of the catalytic fabric can be of any type: simple, mesh, or satin weaving and other possible patterns.

SGFWC systems for cleaning industrial gas emissions do not require a significant modification of the equipment of the process. The catalytic replaceable "cartridge" is made in the form of a cassette element that is easily installed into the reactor-neutralizer and easily extracted from it. The time of installation and dismantling of the catalytic cartridge without stopping the technological process does not exceed $1-2 \mathrm{~h}[11,12]$.

A schematic diagram of a standard industrial process for purification of gas emissions using granular catalysts is shown in Fig. 2. The process is carried out using the equipment purchased from the Oxy France company (France) at the OOO "Plastic" plant (Syzran, Samara oblast). A vapor-air mixture containing solvent vapors (such as methyl ethyl ketone, cyclohexanone, butan-2-one, etc.) is fed into the heating chamber at a temperature of $25-30^{\circ} \mathrm{C}$ by means of a fan, wherein the temperature is substantially higher due to combustion of a propane-butane mixture. In the heating chamber, the vapor-air mixture is heated to $550^{\circ} \mathrm{C}$ and then passed into a catalytic reactor in which the intense oxidation of solvent vapors takes place on the surface of granular catalysts. In our case, the latter are replaced by a cassette assembly containing layers of SGFWC in a certain assortment set and sequence. The standard catalytic reactor has four sec- tions filled with $\sim 2$ tons of granular catalyst. Note that, in these sections, without changing the design of the reactor, the granular catalyst can be replaced by a cassette-design SGFWC. The productivity of this catalytic cleaning system is $25000 \mathrm{~m}^{3} / \mathrm{h}$. Purified hot air enters the heat exchanger, wherein the temperature drops to $300^{\circ} \mathrm{C}$, then flows through a $\sim 40$-m-high column and escapes into the atmosphere. The essence of the catalytic processes for purification from harmful organic substances and carbon monoxide is that the catalyst provides an oxidative or reductive decomposition of toxic impurities to harmless substances, such as water, nitrogen, and carbon dioxide. Nitrogen oxides are reduced to nitrogen.

As an example of the use of our catalysts, we present the characteristics of the SGFWC system used at the Lipetsk STINOL plant to clean the exhaust air from the chambers for painting of refrigerators (the purification system has been in continuous operation for more than 5 years):

- the flow rate of air to be cleaned, $10000 \mathrm{~m}^{3} / \mathrm{h}$;

- the temperature at the catalyst inlet, $350-400^{\circ} \mathrm{C}$;

- the content of impurities (organic solvents), $0.5-1.0 \mathrm{~g} / \mathrm{m}^{3}$;

- degree of purification, 90-95\%;

-woven catalysts are used in the form of a layerby-layer-folded cartridge: the surface area of the catalytic fabric required to completely fill the reactor is $100 \mathrm{~m}^{2}$, with a specific weight of $0.7 \mathrm{~kg} / \mathrm{m}^{2}$; the catalytic cassette is assembled from eight layers with a total assembly thickness of 8-10 $\mathrm{mm}$ (Table 1).

In connection with the above, it should be emphasized that, as compared to traditional granular and powder catalysts, the same quality and volume of air purification is provided by a significantly smaller ( 12-16-fold) volume and weight of SGFWC, and, in addition, it has a longer service life, by a factor of $\sim 1.2-1.5$. The use of SGFWCs also makes it possible to considerably simplify the replacement of the spent catalyst with a new one (one cassette with another, without actually interrupting the technological pro- 
(a)

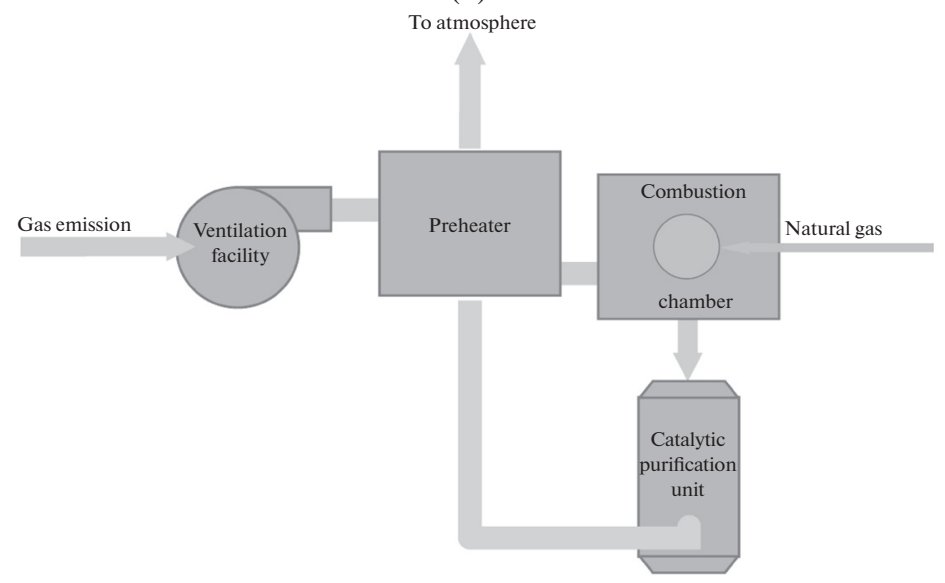

(b)

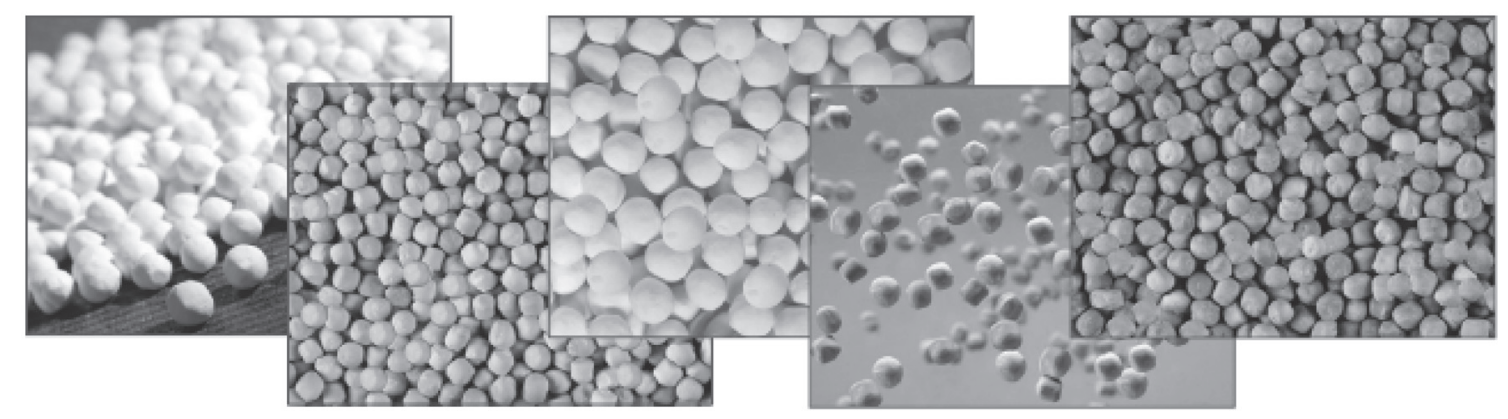

Fig. 2. (a) Schematic diagram of the process of purification of industrial air emissions. (b) Granulated catalysts and $\mathrm{Al}_{2} \mathrm{O}_{3}-$ based supports for use in industrial processes (produced by the Redkinsk catalyst plant), from left to right: high-strength carrier (maximum operating temperature, $\left.T_{\max } \sim 800^{\circ} \mathrm{C}\right)$, a catalyst for high-temperature purification of gas emissions $\left(T_{\max } \sim 500^{\circ} \mathrm{C}\right)$, catalyst with a high thermal conductivity $\left(T_{\max } \sim 1100^{\circ} \mathrm{C}\right)$, catalyst for the removal of hydrogen from the industrial gas cycle at nuclear power plants $\left(T_{\max } \sim 600^{\circ} \mathrm{C}\right)$, and catalyst for use in catalytic combustion (oxidation) and removal of potentially hazardous organic compounds from the gas cycle $\left(T_{\max } \sim 500^{\circ} \mathrm{C}\right)$.

cess, instead of carrying out an expensive unloading-loading procedure in the case of a granular catalyst, which requires a long stoppage of the production process). This makes it possible to reduce the overall operating costs by more than threefold.

\section{NEW APPROACHES IN THE CONTACT STAGE OF NITRIC ACID PRODUCTION}

SGFWC elements with a platinum or palladium content of $0.01-0.1$ wt $\%$ show a high activity in ammonia oxidation, commensurate with the activity of regular catalytic platinoid-based nets, the mass of platinoid in which is approximately three orders of magnitude higher than in SGFWCs. The selectivity of SGFWC systems achieved to date is somewhat lower than that of platinum-based metal grids (85-90\% vs. $95 \%$ ), a factor that currently allows the use of SGFWC cassettes in ammonia conversion reactors only in the form of a combination with platinoid-based nets. However, even this scheme allows to reduce the mass of the platinoids in the catalytic package of the reactor by $20-50 \%$ and to reduce their losses twofold $(0.06 \mathrm{~g}$ of platinum per ton of produced acid versus the nominal index of $0.12 \mathrm{~g} /$ ton). This structure of the catalyst package is currently being used in industrial reactors of some Russian enterprises producing nitric acid. There is reason to expect that, in the near future, SGFWC packages will replace traditional platinoid-based nets, which are operated in the production of nitric acid without alternative, in essence, throughout its history. To solve this fundamental problem, it is necessary to improve two parameters of SGFWC: to raise the selectivity from $85-90 \%$ to $95 \%$ and to increase the operational thermal resistance from 750 to $850^{\circ} \mathrm{C}$. Developers have scientific and technological approaches to improve the indicated characteristics of the SGFWC [13-15].

\section{USE OF SGFWC IN PRODUCTION OF SULFURIC ACID}

As a result of the research conducted by the current team of authors, it was established that SGFWC elements doped with platinum to $\sim 0.05$ wt $\%$ exhibit a catalytic activity significantly exceeding that of vanadium catalytic systems, traditionally used in this pro- 
cess. The changeover to SGFWCs in the production of sulfuric acid makes it possible to reduce the contact temperatures by $40-60^{\circ} \mathrm{C}$, which, in turn, leads to an increase in the degree of conversion of sulfur dioxide and to a reduction in its content in gas emissions; i.e., one of the most important environmental problems in the technology of sulfuric acid production is solved.

Efforts to introduce SGFWCs in the technology of sulfuric acid production instead of the traditional granulated bulk vanadium catalysts have been undertaken by the authors in recent years together with established producers of sulfuric acid, such as "Voskresensk Mineral Fertilizers" (Voskresensk) and "FosAgro-Cherepovets" (Cherepovets), as well as with Samoilov Research Institute of Fertilizers and Insectofungicides, a leading developer of technological processes in acid production (Moscow).

In the currently implemented program of scientific and technological search, the following main stages can be distinguished:

(1) Performing laboratory tests to study the effectiveness of various modifications of SGFWCs in comparison with vanadium catalysts currently used at domestic enterprises (produced by the BASF and other foreign companies);

(2) Resource testing SGFWCs by placing them directly into the granulated vanadium catalyst bed in an industrial reactor.

(3) Conduction of technological studies on the conversion of $\mathrm{SO}_{2}$ into $\mathrm{SO}_{3}$ and optimization of the operational parameters using a pilot reactor with SGFWC under typical industrial conditions, more specifically, installed in parallel to the fourth shelf of an industrial reactor. At the OAO "Voskresensk Mineral Fertilizers" enterprise, for example, a two-stage scheme with an intermediate absorption is used, in which three shelves of the first-in-sequence reactor are loaded with a vanadium catalyst, whereas in the second, the fourth and fifth shelves are loaded.

(4) Organization of industrial tests of SGFWCs by loading one of the shelves of an industrial reactor with layer-by-layer-folded elements of a glass-fiber catalyst instead of a bulk vanadium catalyst.

To date, the realization of the above program has led to the following results. Laboratory and industrial tests of various SGFWC samples in sulfur dioxide oxidation reactions have shown that these catalytic materials really represent an effective alternative to traditional vanadium catalysts regarding a number of parameters. We carried out standard test evaluations of the activity of catalysts in the processes of sulfur dioxide conversion under the following conditions: the content of platinum in the SGFWC, $0.05-0.2 \mathrm{wt} \%$; the concentration of $\mathrm{SO}_{2}$ in the reaction flow, is $10 \mathrm{vol} \%$; and the flow temperature at the inlet, is $350-500^{\circ} \mathrm{C}$. As a result of a full cycle of experiments, it was found that SGFWCs can be characterized as the best of all known industrial catalysts, including those produced by Monsanto (LR-120, LP-110; USA), BASF (04-110,
04-111; Germany), Haldor Topsoe (VK-38, VK-48; Denmark), and Catalyst Chemical Europe (C-116-3, C-116-3-03, C-116-3HYA). Silica glass-fiber catalysts are characterized by a high activity (especially at low temperatures, with comparative tests performed at 420 and $485^{\circ} \mathrm{C}$ ), low "ignition" temperature of the process $\left(350-380^{\circ} \mathrm{C}\right.$, depending on the internal surface of the catalyst sample), and a significantly higher thermostability $\left(700-750^{\circ} \mathrm{C}\right)$ as compared with vanadium catalysts (this factor is especially important for the production of sulfuric acid at nonferrous metallurgy enterprises).

Resource tests of glass-fiber catalysts were carried out by placing SGFWC samples directly into the granular vanadium catalyst bed of an active industrial reactor. It was found that, during an annual run, the samples did not lose their catalytic activity, remaining mechanically undestroyed.

To date, most of the pilot testing program has been implemented on a model reactor with a diameter of $300 \mathrm{~mm}$, connected in parallel to the fourth shelf of a second-stage industrial reactor, i.e., under conditions as close to actual production as possible. The test parameters were as follows: the concentration of sulfur dioxide in the reaction stream was 9 vol $\%$, the inlet temperature in the catalytic bed was $380-450^{\circ} \mathrm{C}$, the content of platinum in the SGFWC samples was $0.10-0.15 \mathrm{wt} \%$, the number of layers in the SGFWC package was 20-60 (the total height of the cassette was $15-50 \mathrm{~mm})$, and the flow velocity was $0.13 \mathrm{~m} / \mathrm{s}$. The results of the comparative pilot tests are listed in Table 2. This table shows the advantages of SGFWCs in comparison with the vanadium catalyst: (1) the contact time is reduced by an order of magnitude, (2) the height of the catalyst lays is 20-30 times smaller, and (3) it is obvious that the cassette-type design of SGFWCs provides significant advantages in operating the reactor. The economic assessments show that the cost of a SGFWC package will be $20-50 \%$ lower than the cost of loading the reactor with traditional vanadium catalysts $[16,17]$.

\section{SGFWC-ASSISTED “CATALYTIC" NITRIDING: NEW PERSPECTIVES OF 100-YEAR TECHNOLOGY OF CHEMOTHERMAL TREATMENT OF THE SURFACE OF METAL ITEMS}

The scientific basis underlying the development of the technologies discussed in this section is a concept nonconventional for the currently existing theory of nitriding and hardening. It is based on the hypothesis that active gaseous species in the nitriding of metals active are not the stable equilibrium products of interaction of the furnace atmosphere components, but intermediate, labile, chemically highly reactive species, such as nitrogen-, hydrogen-, and oxygen-containing radicals, as well as ions and ion radicals. This hypothesis led the current authors to examine the expediency of introducing into the process a catalytic 
Table 2. Comparative characteristics of the SGFWC systems and traditional granular $\mathrm{V}_{2} \mathrm{O}_{5}$-based catalysts in the processes of sulfuric acid production

\begin{tabular}{l|c|c|c|c|c}
\hline \multicolumn{1}{c|}{ Reactor type } & $\begin{array}{c}\text { Temperature, } \\
{ }^{\circ} \mathrm{C}\end{array}$ & $\begin{array}{c}\text { Bed resistance } \\
\mathrm{mmH}_{2} \mathrm{O}\end{array}$ & $\begin{array}{c}\text { Degree } \\
\text { of conversion, } \%\end{array}$ & $\begin{array}{c}\text { Catalyst bed height, } \\
\mathrm{mm}\end{array}$ & $\begin{array}{c}\text { Contact time, } \\
\mathrm{s}\end{array}$ \\
\hline $\begin{array}{l}\text { Pilot SGFWC-filled } \\
\text { reactor }\end{array}$ & $420-430$ & 32 & $59-62$ & $15-20$ & $0.12-0.15$ \\
\hline $\begin{array}{l}\text { Industrial reactor } \\
\text { (V-catalysts) }\end{array}$ & $420-430$ & 32 & 59 & 450 & 1.5 \\
\hline
\end{tabular}

factor, never before employed in the practice of nitriding steels and alloys, a factor capable of selectively affect the transformation of the ammonia-air medium during its interaction with metallic surfaces.

The results of these studies fully confirmed the validity of the initial assumptions: the nitriding process radically changed its character in the regime parameters and in the quality of the processed item. Upon installation of a catalytic reactor equipped with a SGFWC cassette assembly on the ammonia or ammonia-air line of the nitriding furnace provided a radical activation of the furnace atmosphere, which manifested itself through the following features of the nitriding of metal surfaces [18, 19]:

- the corrosion resistance of structural steels was significantly increased;

- the hardness of the processed product was increased to a depth of more than $1 \mathrm{~mm}$;

- the wear resistance and hardness of the treated item increased;

- the process of decarbonization during nitriding was suppressed;

- the process of nitriding was substantially accelerated of (hours instead of days); times;

- the consumption of ammonia was reduced many

-new practical applications of the proposed technology to nitriding materials non-nitrideable by the traditional noncatalytic technology, such as highspeed steels, furnace metallurgy products, titanium and its alloys, have been found.

The technology was developed on the industrial scale at a number of Russian metallurgical and engineering enterprises [20]. A classic example of equipment for the implementation of catalytic gas nitriding in a shaft furnace is the CCHV 6.9/7 furnace and its analogues produced by ZAO "Nakal" (Solnechnogorsk) (Fig. 3).

The shaft furnace for gas nitriding is equipped with a device for catalytic treatment of articles in an ammonia-nitrogen atmosphere, which ensures the saturation of iron, steel, and alloys with nitrogen under nearly equilibrium conditions. Individual SGFWC elements or a SGFWC cassette assembly can be placed inside this device in any number and in any form necessary to implement a particular nitriding process. In order to take into account the influence of certain variables, a system of indirect control of the nitriding potential of the furnace atmosphere was developed. With a certain content of nitrogen in the surface layers of the workpiece, this system allows the operator to change in real time the flow rate of process gases, as well as the temperature and dynamics of diffusion. The system also makes it possible to calculate the distribution of nitrogen concentration from the surface to the bulk of the product, the phase composition of the near-surface zone, and the microhardness distribution in the diffusion layer (Fig. 4).

The technology of catalytic nitriding in SGFWC reactors with catalysts of various designs has been applied in practice for processing a wide range of articles: gear wheels; shafts and rods; parts of pumps; hydraulic and pneumatic equipment; stamps and molds; metalware with an anticorrosive coating made of various materials, such as structural steels, cemented steels with a combination of nitriding and quenching, die-cast alloys, cold-deforming steels, high-speed steels, stainless steels; products of powder metallurgy, as well as products made of titanium and its alloys.

\section{NEW SOLUTIONS IN HYDROCARBON HYDROGENING TECHNOLOGY}

To date, laboratory and pilot-plant tests have yielded results indicative of wide prospects for the use of SGFWC elements doped with palladium (up to $0.1 \mathrm{wt} \%$ ) in the hydrogenation of hydrocarbons. As an example of the efficiency of SGFWC in gas-phase hydrogenation, it is worthwhile to mention works on studying the purification of synthesis gases in the production of olefins and synthetic rubber monomers from acetylenic compounds [21, 22]. Fundamental changes in traditional technologies should be expected from application of SGFWCs to liquid-phase hydrogenation of hydrocarbons, especially in the hydrogenation of natural oils and fats of vegetable and animal origin, as well as in the reduction of nitroaromatic compounds to amines.

\section{Hydrogenation of Vegetable Oils on SGFWCs}

The developed technology can be successfully applied at margarine industry enterprises at the stage 
(a)

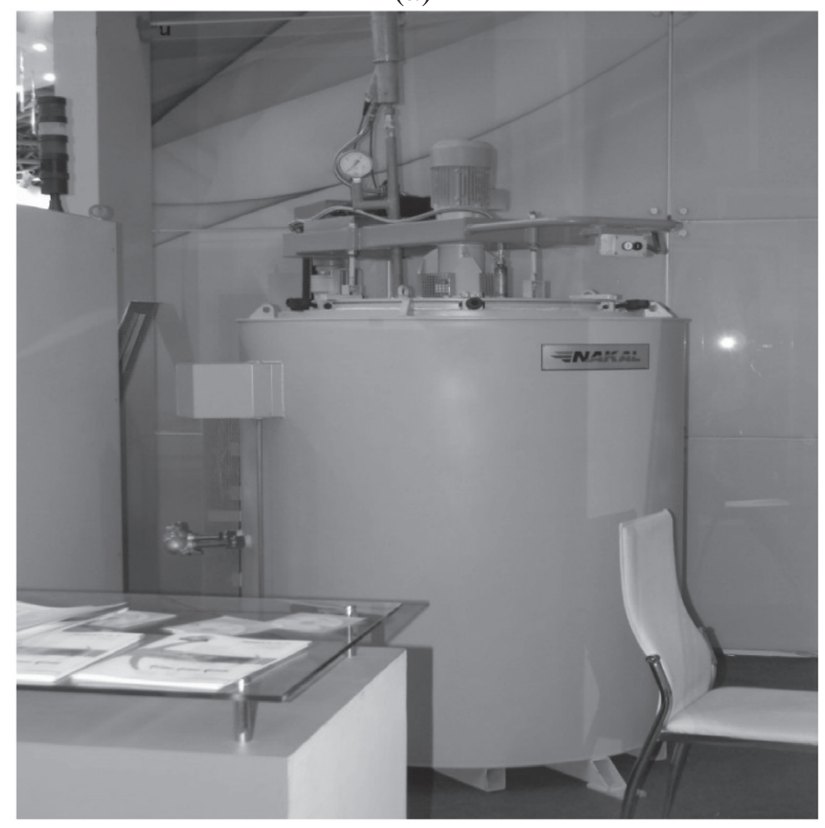

(b)

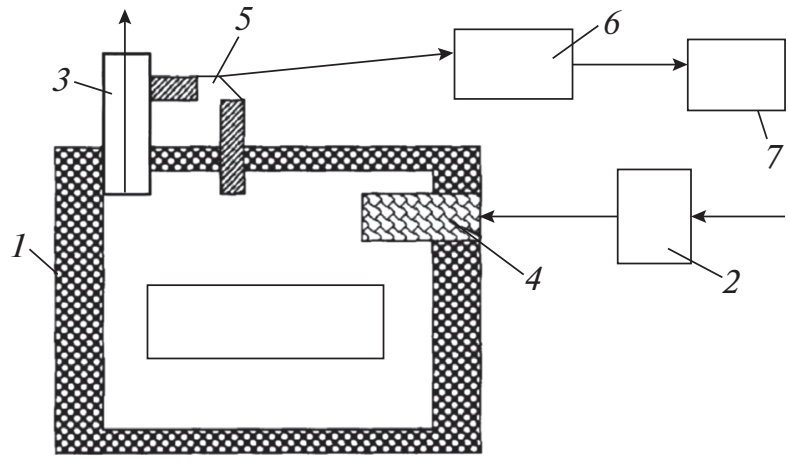

Fig. 3. (a) General view of a shaft furnace for gas-phase nitriding (supplier, ZAO "Nakal", Solnechnogorsk). (b) Schematic diagram of a shaft furnace for catalytic gasphase nitriding (working chamber dimensions: $D \sim 600 \times$ $\left.900 \mathrm{~mm}, H \sim 1000 \times 2000 \mathrm{~mm} ; T_{\max } \sim 700^{\circ} \mathrm{C}\right) ;(1)$ heating furnace; (2) feeding, mixing, and portioning device; (3) device for the removal of process gases driven from low pressure lines; (4) unit for producing a catalytic effect on the furnace atmosphere; (5) oxygen sensor (device for indirect monitoring and control of the nitrogen potential of the furnace atmosphere); (6) secondary converter with indication of the nitrogen potential (weight content of nitrogen in iron); and (7) computer control device (automatic or through the operator).

of hydrogenation of natural oils in order to obtain solid fats. The use of SGFWC in this process makes it possible to abandon the traditional energy-intensive, environmentally hazardous process schemes for the hydrogenation of vegetable oils, based on the use of finely dispersed suspended nickel catalysts. The SGFWC systems offer fundamentally new technological solutions for this process and nontraditional schemes of reactor design, providing a complete rejec- (a)

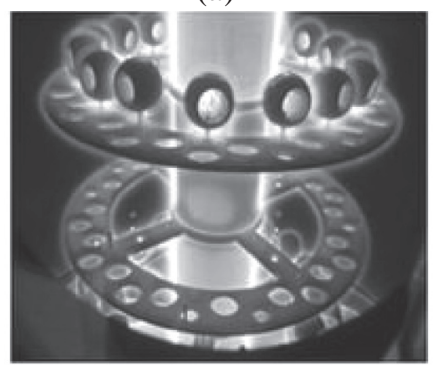

(b)

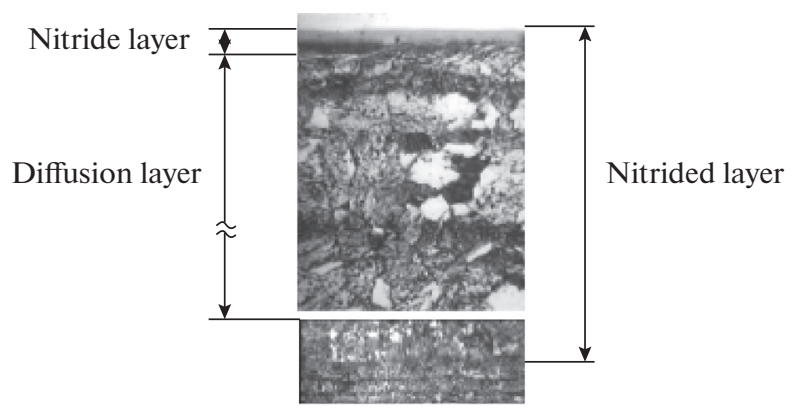

(c)

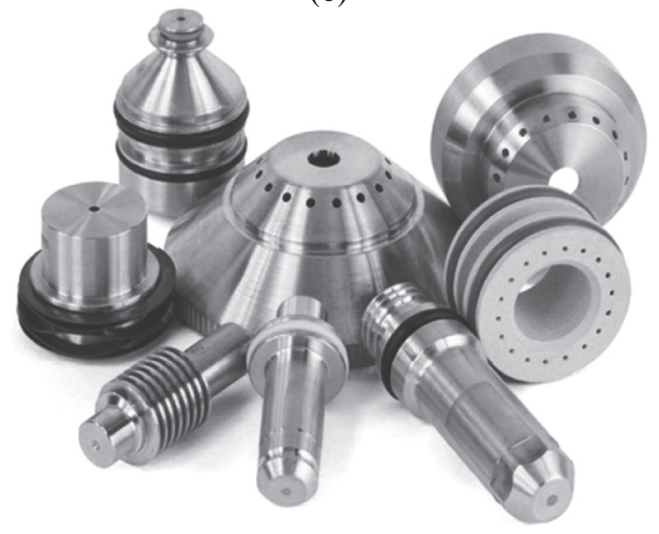

Fig. 4. (a) Process of high-temperature nitriding of parts in a furnace (picsbase.ru). (b) Nitrogen concentration distribution from the surface of the steel part to the interior; microsection of the nitrided part (magnification 500×, tehsovet.ru, N2(44), 15.02.2007). Traditional technologies are characterized by a $\sim 0.1-\mathrm{mm}$ nitride layer, $1.0-\mathrm{mm}$ diffusion layer, and $1.5-\mathrm{mm}$ nitrided layer. With the use of catalytic nitriding (by SGFWC), the nitride layer thickness is increased to $1 \mathrm{~mm}$. (c) General view of the parts after completion of the nitriding process (OAO "Pnevmostroymashina", Yekaterinburg).

tion of the extremely expensive stages of filtration purification of the resultant hydrogenated fat from the catalyst and a reduction in the residual content of the metal product by an order of magnitude.

The technology of the process and its hardware design were worked out in pilot tests. According to the proposed technology, the hydrogenation of vegetable oils and fats is carried out by hydrogen fed into a reactor with a screw stirrer. The catalytic fabric is fixed on 
a cylindrical frame, with the agitator screw inside. During operation, the catalytic "cartridge-diffuser" formed in this way is located in the hydrogen-saturated liquid medium circulating inside the reactor. The proposed design of the catalyst placement unit is easy to implement in existing reactors for the production of hydrogenated fat without noticeable expenditures for their reconstruction [23]. Obviously, the time required to install and dismantle the catalyst diffuser-cartridge described above is extremely small. The reactor of the proposed design can operate in both the batch and continuous modes. Unlike hydrogenation on powder catalysts, widely used in the margarine industry, which involves expensive, energy-intensive, and difficult-tooperate filtration-mode purification of the product from the catalytic powder, the proposed glass-fiber catalyst technology is free of the filtration operation.

The use of glass-fiber catalysts in the production of solid vegetable oils and fats, as an alternative to the traditional process of liquid-phase hydrogenation on $\mathrm{Ni}$ containing powder catalysts, provides the following advantages [24]:

-exclusion from the process the energy-intensive stage of deep filtration of the product to clean it from the powder components and the entailing reduction in the cost of the fat by $20-30 \%$;

- a complete elimination of nickel from the final product, a heavy, physiologically dangerous metal;

- the possibility of performing the hydrogenation of oils in the continuous mode, which significantly enhances the productivity of existing equipment without additional investments;

- the elimination of the environmentally sensitive issue of disposal of large amounts of Ni-containing spent catalysts accumulated at the enterprises producing hydrogenated fat, which require deep and expensive processing $[24,25]$.

\section{Reduction of Nitroaromatic Compounds on SGFWCS}

The authors have thoroughly investigated the possibilities of using SGFWCs doped palinodes in the liquid-phase hydrogenation of mono- and polyfunctional aromatic nitro compounds (from mono- to trinitrobenzene and trinitrotoluene) instead of traditional powdered-carbon-supported palladium catalysts [26-28]. As a result, it was found that glass-fiber catalysts are characterized by an activity significantly exceeding that of conventional powder systems. An important result of the studies was the discovery of an extremely high selectivity of these systems with respect to the sequential hydrogenation of nitro groups (in the $\mathrm{Pd} / \mathrm{C}$-powder system, the reduction proceeds synchronously for all nitro groups, practically nonselectively). Even now, the practical importance of these studies is obvious, which opens the possibility for a onestage synthesis of complex nitroamines. Moreover, this process should be considered as the technological basis for the development of schemes for the conversion of nitroaromatic explosives (in particular, TNT) into useful civilian products.

Based on the results of our studies, we proposed a method for the chemical utilization of demilitarized explosives, for example 2,4,6-trinitrotoluene (TNT), by converting it into 2,4,6-triaminotoluene (TAT) (Fig. 5). The latter can be used manufacture competitive products and materials with a valuable set of properties important for practice, for example, $N^{1}, N^{1}, N^{2}, N^{2}, N^{3}, N^{3}$-hexa(2-hydroxypropyl)-2,4,6-triaminotoluene (HOPCAT), a unique "stitcher" for polyurethane foams. There is a number of other products, for example, an effective fire extinguishing composition based on the action of a foamed product of TAT condensation with formaldehyde, a technical product methylphloroglucinol, a compound with a wide range of uses, and 4-hydroxy-2,6-diaminotoluene, a new stable monomer (diamine) with a free hydroxyl group. In addition, new environmentally friendly closed-cycle technologies for manufacturing these articles and materials for its production have been developed, which can be realized at atmospheric pressure and low temperature (up to $100^{\circ} \mathrm{C}$ ) with the use of water as a reaction medium.

In comparison with the traditional technologies, the proposed methods for the catalytic reduction of aromatic nitro compounds to the corresponding amines have the following advantages:

- The possibility to carry out three-phase catalytic processes in compact and efficient CGFC-filled reactors of new type, without traditional powder catalysts and the entailing filtration stage. This will ensure a high specific rate of the process and, as a result, a significant simplification of the process of hydrogenation of aromatic nitrocompounds and accordingly, a reduction in total capital investments and operation costs.

- The use of a continuous automated technological cycle of production and regeneration of catalysts, which provides flexibility in changing the operation mode of the process and the possibility of preparing catalysts with a high degree of reproducibility and significantly simplifies the technology.

\section{USE OF SGFWCS IN THE PROCESSES OF CATALYTIC PURIFICATION OF WATERS FROM NITRATE-NITRITE POLLUTIONS}

Silica fiberglass catalysts have been successfully used in the processes of neutralization of nitrites and nitrates dissolved in water by their reduction in the presence of hydrogen (hydrodenitrification of water). The problem of denitrification of water (especially drinking water) is of great environmental importance and is becoming especially acute nowadays across the world. This is due to the intensive use of nitrogen-containing mineral fertilizers in agriculture, which penetrate into groundwater and natural and artificial water bodies. The concentration of nitrates in these waters 


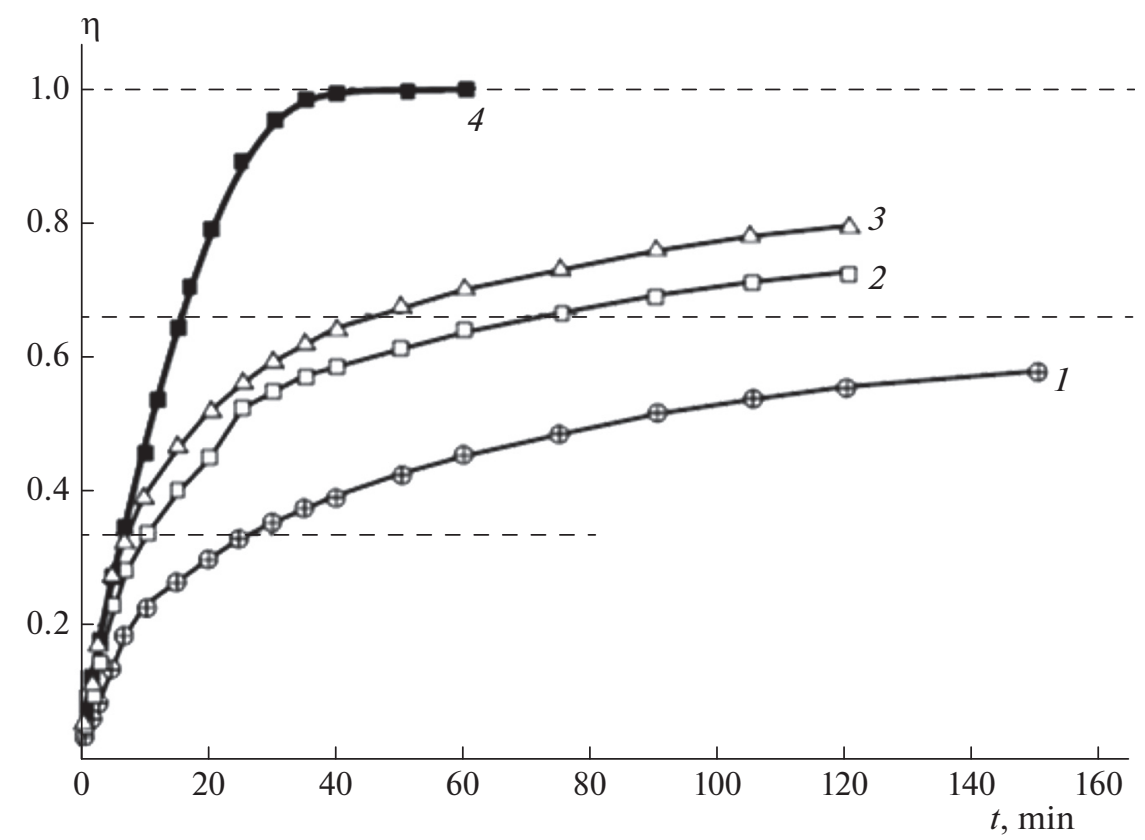

Fig. 5. Comparative dependence of the degree of hydrogen conversion $\eta$ on the transformation time $t$ for the hydrogenation of TNT on a SGFWC with a content of $0.1 \mathrm{wt} \% \mathrm{Pt}$ and specific surface areas $\left(\mathrm{m}^{2} / \mathrm{g}\right)$ of (1) 1 , (2) 5, and (3) 15 and (4) on a 5\% Pd/C powder catalyst $\left(500 \mathrm{~m}^{2} / \mathrm{g}\right)$.

has increased significantly in recent years and has reached the critical level in many regions to date.

Nitrate and nitrites are stable, readily soluble salts, poorly adsorbed and incapable of coprecipitation. These properties of these salts create fundamental difficulties for their removal from water by such a traditional method of treating aqueous media as softening by liming and subsequent filtration. Various physicochemical and biological methods of water denitrification, such as ion exchange technologies, osmotic separation methods, electrodialysis methods, water treatment processes with various biologics, are expensive and involve the use of significant masses of solid components to be removed from the purified water. The situation is also complicated because of the need to introduce into the technological scheme long and costly stages of regeneration of these solid components.

The method of denitrification of water by catalytic reduction of nitrates and nitrites is recognized as the most effective, easy-to-use and economically advantageous among other known methods, allowing at the same time to ensure $100 \%$ purification of water from these impurities. The process is based on the use of metal-containing catalysts on a support, on which, with the participation of hydrogen, nitrates and nitrites dissolved in water are reduced to nitrogen.

The most common form of water denitrification catalysts adopted in practice are systems based on powdered supports (carbon, silica gel, or alumina gel), which are present in the aqueous medium to be purified in a suspended state. Obviously, such systems are characterized by significant technological and opera- tional shortcomings. Firstly, the process in which a powdered suspended catalyst is used is difficult to control. Secondly, a powdered catalyst requires the use of a reactor equipped with stirring devices to form a suspended reaction medium and to speed up the process of diffusion, which can potentially limit the denitrification rate. Thirdly, the use of a powdered suspended catalyst is associated with the need to introduce into the process the stage of filtration to remove catalyst particles from the purified aqueous media. The presence of filtration stages in the scheme requires involves considerable energy consumption and entails a technologically unprofitable periodical mode of operation.

The studies performed of the denitrification of waters on SGFWCs showed that the transfer of the technological scheme to the use of glass-fiber woven catalysts makes it possible to radically improve the characteristics of the process and eliminate the abovementioned disadvantages typical of the denitrification on traditional suspended powder catalysts [29-31].

We tested a wide variety of porous SGFWC samples prepared from silica and borosilicate glass in the form of woven fabric or nonwoven blocks and having an internal specific surface area of $2-100 \mathrm{~m}^{2} / \mathrm{g}$. The active metal was a platinoid (palladium, platinum, rhodium, etc.), in some cased modified by other metals, such as copper, tin, silver, etc. The total metal content in the fibrous carrier varied from 0.01 to $1.0 \mathrm{wt} \%$.

Silica glass-fiber catalysts can be used in the processes of water denitrification over a wide range of nitrate (nitrite) concentrations. The process of neutralization of nitrates and nitrites is optimized depend- 
ing on the following factors: the initial nitrate (nitrite) concentration, $\mathrm{pH}$ of the treated medium, hydrogen feed rate, and temperature. The process of denitrification on the proposed catalyst occurs at an appreciable even at temperatures of $5-30^{\circ} \mathrm{C}$.

Summarizing the obtained experimental results led us to the following conclusions on the effectiveness of the proposed SGFWC technology:

- The new technology of water denitrification is based on the reduction of nitrates and nitrites on SGFWCs, a novel class of catalysts for this technology. These catalysts are highly effective systems, stably operating at temperatures of $10-30^{\circ} \mathrm{C}$ and $\mathrm{pH} 6-7$ (for example, 40 times as effective as biological denitrification).

- The technology is very simple to operate and makes it easy to restore bound nitrogen to the molecular, reducing the concentration of the former from 100 to $2-5 \mathrm{mg} / \mathrm{L}$.

-The new technology has significant operational, technological, and economic advantages over other the traditional processes of water denitrification, including osmotic, ion exchange, and biological methods: the possibility of implementing continuous denitrification schemes, eliminating the stages of filtering off the dispersed catalyst from purified water.

\section{CATALYTIC HEAT AND GAS GENERATORS}

SGFWC elements doped with platinum (up to $0.01-0.1 \mathrm{wt} \%$ ) or chromium exhibit high catalytic activity in the reactions of deep oxidation of hydrocarbons. The analysis of the efficiency of their use in catalytic heat generators for industrial and household purposes testifies to significant advantages of SGFWCs over traditional ceramic catalytic burners: cheapness; simplicity of assembling, installation, and replacement of the catalytic element; and resistance to vibrational and shock loads. At present, the first prototypes of catalytic heaters with SGFWC elements have been manufactured. Information obtained in the course of laboratory and pilot industrial-scale tests of the performance of SGFWCs in the flameless combustion of gaseous and liquid hydrocarbon fuels led us to conclude that these catalytic systems are promising in solving a variety of problems important for modern power engineering and engine building, such as the transfer of gas turbines from torch burning to a lowtemperature, flameless catalytic combustion of fuel, characterized by a lower heat loss and the absence of nitrogen oxides in the exhaust gas.

\section{NEW SORBENTS BASED ON FIBERGLASS WOVEN MATERIALS}

Fundamental studies of the mechanism and kinetics of ion-exchange processes in silicate glass-fiber woven matrices carried out in works on the problems related to SGFWCs, led to the creation of a new gen- eration of wide-purpose sorbents. Glass-fiber woven sorbents have significant advantages over their conventional analogues, such as granular powdered silica gels and alumogels, in a number of important parameters, in particular, in the controllability of product characteristics, range of parameters, scope of possible applications, sorption capacity of the absorber and its saturation dynamics, and variety of types and structures of sorbent products. Woven glass-fiber sorbents are designed for:

—operation as desiccants of gas flows;

—purification of gas emissions from organic and inorganic impurities;

-carrying out the processes of ion exchange and purification of effluents from ions of heavy metals and other contaminants;

-sorption-mode separation, concentration, and extraction of valuable components from liquid wastes;

- use as dehumidifiers and absorbers in refrigerators, air conditioners, and other household devices;

- use as carriers of catalysts, enzymes, and other functional groups in biochemistry and biotechnology;

- use for analytical purposes.

Currently, preparations are being made for launching the industrial production of glass-fiber woven sorbents, which are characterized by the following values of some technical parameters [32]:

-internal specific surface area, 1-100 $\mathrm{m}^{2} / \mathrm{g}$;

- pore diameter, 10-200 ̊;

-adsorption capacity for water at drying, $30 \mathrm{wt} \%$;

capacity for benzene adsorption from the gas phase, 25 wt \%;

- the diameter of the elementary fiber in the fabric, 5-10 $\mu \mathrm{m}$;

—product form: canvas, satin weave, woven blocks.

\section{CONCLUSIONS}

The results of the development of SGFWC materials and their practical applications in a wide variety of technological processes considered in the present review have been obtained only due to a multilateral cooperation of scientific research institutes and industrial enterprises. Because of this cooperation, it became possible to conduct practical tests of SGFWCs in various forms, designs, and aggregates, with doping them by various metals, as an alternative to currently used powdered and granular catalysts. This include the use of SGFWC elements for purification of gas emissions and wastewater of industrial enterprises from organic pollutants, in various stages of sulfuric and nitric acid production; in the processes of "catalytic nitriding" of metal surfaces; in the hydrogenation of vegetable, animal, and artificial oils and fats; in catalytic heat generators; and as glass-fiber woven sorbents for various applications. This new technology is a real alternative to the current technological approaches based on the use of 
powdered and granular catalysts, due to a low cost, simplicity of manufacture and operation, and simplicity and cheapness of replacement and disposal. In addition to purely technological advantages, the results obtained create a basis for searching for new approaches to the synthesis and development of new types of materials for adsorption and catalysis, as well as for the production of new equipment for catalytic processes.

The corporation established for research and solving problems related to SGFWCs, including Institute of Problems of Chemical Physics of the Russian Academy of Sciences (IPCP, Chernogolovka), OOO "Khimfist" (Chernogolovka), DMF "Stekloplastik" (Kryukovo), IA "Steklovolokno" (Polotsk, Republic of Belarus), Institute of Catalysis (Siberian Branch of the Russian Academy of Sciences, Novosibirsk), FSUE "Moscow Special Alloys Plant" (Moscow), OAO "ScientificResearch Institute for the Technology of the Automotive Industry" (Moscow), OAO "STINOL" (Lipetsk), OAO "Voskresensk Mineral Fertilizers" (Voskresensk), OAO "Khimkombinat" (Kirovo-Chepetsk), OAO "Minudobreniya" (Rossosh), OAO "AKRON" (Veliky Novgorod), "Technion" (Haifa, Israel) and others, is continuing its fruitful cooperation in the development of this promising scientific-technological direction.

\section{ACKNOWLEDGMENTS}

I. Parkin is grateful to the Royal Society (UK) for support.

This work was supported by the Russian Foundation for Basic Research, project nos. 13-03-12407 ofi_m2 and 13-03-12412 ofi_m2).

\section{REFERENCES}

1. Ch. Krauns, V. Barelko, G. Fabre, et al., Catal. Lett. 72, 161 (2001).

2. L. G. Simonova, V. V. Barelko, O. B. Lapina, E. A. Paukshtis, V. V. Terskikh, V. I. Zaikovskii, and B. S. Bal'zhinimaev, Kinet. Catal. 42, 693 (2001).

3. L. G. Simonova, V. V. Barelko, BE. A. Paukshtis, O. B. Lapina, V. V. Terskikh, V. I. Zaikovskii, and B. S. Bal'zhinimaev, Kinet. Catal. 42, 828 (2001).

4. L. G. Simonova, V. V. Barelko, A. V. Toktarev, V. I. Zaikovskii, V. I. Bukhtiyarov, V. V. Kaichev, and B. S. Bal'zhinimaev, Kinet. Catal. 42, 837 (2001).

5. T. Gotoh, S. Nakamura, J. Abe, et al., US Patent No. 4038214 (1977).

6. O. Nakamura, Jpn. Patent No. 22145 (1980).

7. J. Yagi, H. Nakamura, and M. Fukai, Jpn. Patent No. 137752 (1988).

8. V. V. Barelko, B. S. Bal'zhinimaev, S. P. Kil'dyashev, et al., RF Patent No. 2143948, Byull. Izobret. No. 1 (2000).

9. V. V. Barelko, I. A. Yuranov, A. F. Cherashev, A. P. Khrushch, V. A. Matyshak, T. I. Khomenko, O. N. Sil'chenkova, and O. V. Krylov, Dokl. Phys. Chem. 361, 233 (1998).

10. I. Yuranov, L. Kiwi-Minsker, V. Barelko, et al., in Reaction Kinetics and Development of Catalytic Pro- cesses, Ed. by G. F. Froment and K. C. Waugh (Elsevier Science B.V., Amsterdam, 1999), p. 191.

11. V. V. Barelko, A. A. Prudnikov, L. A. Bykov, et al., RF Patent No. 2171430, Byull. Izobret. No. 21 (2001).

12. V. V. Barelko, A. P. Khrushch, A. F. Cherashev, I. A. Yuranov, V. A. Matyshak, O. N. Sil'chenkova, T. I. Khomenko, and O. V. Krylov, Kinet. Catal. 41, 655 (2000).

13. V. V. Barelko, P. I. Khal'zov, V. Ya. Onishchenko, et al., RF Patent No. 2069584, Byull. Izobret. No. 33 (1996).

14. V. V. Barelko, P. I. Khal'zov, V. Ya. Onishchenko, et al., RF Patent No. 2069585, Byull. Izobret. No. 33 (1996).

15. V. V. Barelko, V. Ya. Onishchenko, B. S. Bal'zhinimaev, et al., RF Patent No. 2160157, Byull. Izobret. No. 34 (2000).

16. L. G. Simonova, B. S. Bal'zhinimaev, S. P. Kil'dyashev, et al., RF Patent No. 2158633, Byull. Izobret. No. 31 (2000).

17. L. G. Simonova, V. V. Barelko, A. V. Toktarev, A. F. Chernyshov, V. A. Chumachenko, and B. S. Balzhinimaev, Kinet. Catal. 43, 61 (2002).

18. V. M. Zinchenko, V. Ya. Syropyatov, V. V. Barelko, et al., Metalloved. Term. Obrab. Met., No. 7, 7 (1997).

19. V. V. Barelko, A. P. Khrushch, A. F. Cherashev, I. A. Yuranov, V. A. Matyshak, O. N. Sil'chenkova, T. I. Khomenko, and O. V. Krylov, Kinet. Catal. 41, $655(2000)$

20. V. Ya. Syropyatov, V. V. Barelko, and V. M. Zinchenko, RF Patent No. 2109080, Byull. Izobret. No. 11 (1998).

21. B. S. Bal'zhinimaev, V. V. Barelko, A. P. Suknev, E. A. Paukshtis, L. G. Simonova, V. B. Goncharov, V. L. Kirillov, and A. V. Toktarev, Kinet. Catal. 43, 542 (2002).

22. V. V. Barelko, A. A. Fomin, S. I. Serdyukov, et al., RF Patent No. 2164814, Byull. Izobret. No. 10 (2001).

23. V. V. Barelko, I. A. Yuranov, A. A. Fomin, et al., RF Patent No. 2109039, Byull. Izobret. No. 11 (1998).

24. V. V. Barelko, P. I. Khal'zov, and S. M. Baturin, RF Patent No. 2081898, Byull. Izobret. No. 17 (1998).

25. V. V. Barelko, B. S. Bal'zhinimaev, S. P. Kil'dyashev, et al., RF Patent No. 2158632, Byull. Izobret. No. 31 (2001).

26. V. G. Dorokhov, V. V. Barelko, B. S. Bal'zhinimaev, et al., Khim. Prom-st., No. 8, 44 (1999).

27. Wu Chuntiag, V. G. Dorokhov, G. A. Boiko, B. S. Bal'zhinimaev, and V. V. Barelko, Dokl. Chem. 402, 111 (2005).

28. V. G. Dorokhov, V. V. Barelko, B. S. Bal'zhinimaev, et al., RF Patent No. 2156654, Byull. Izobret. No. 27 (2000).

29. Yu. Matatov-Meytal, V. Barelko, I. Yuranov, et al., Appl. Catal., B: Environ. 27, 127 (2000).

30. Yu. Matatov-Meytal, V. Barelko, I. Yuranov, et al., Appl. Catal., B: Environ. 31, 233 (2001).

31. V. V. Barelko, I. A. Yuranov, M. Sheintukh, et al., RF Patent No. 2133226, Byull. Izobret. No. 20 (1999).

32. V. V. Barelko, N. P. Kuznetsova, B. S. Bal'zhinimaev, et al., RF Patent No. 2169612, Byull. Izobret. No. 18 (2001). 